\title{
A Fuzzy C-means Model Based on the Spatial Structural Information for Brain MRI Segmentation
}

\author{
Shunfeng Wang ${ }^{1}$, Zhiyuan Geng ${ }^{1}$, Jianwei Zhang ${ }^{1}$, Yunjie Chen ${ }^{1}$ and Jin Wang ${ }^{2}$ \\ ${ }^{1}$ School of Mathematics \& Statistics, Nanjing University of Information \\ Science \& Technology, Nanjing 210044, China \\ ${ }^{2}$ School of Computer \& Software, Nanjing University of Information \\ Science \& Technology, Nanjing 210044, China
}

\begin{abstract}
Due to the effect of noise in brain MR images, it is difficult for the traditional fuzzy c-means (FCM) clustering algorithm to obtain desirable segmentation results. Combining the information of patch to reduce the effect of noise has been a focus of current research. However, the traditional patch model is isotropic, so that it would lose the structural information easily. In this paper, a novel fuzzy c-means model based on the spatial similarity information is proposed. To be anisotropy and preserve more structural information, this model takes both the non-local information and spatial structural similarity measurement (SSIM) between the image patches into consideration, and then a new distance function is established between every pixels and category centers for image segmentation. The efficiency of the proposed algorithm is demonstrated by extensive segmentation experiments using both synthetic and real brain MR images and by comparison with other state of the art algorithms.
\end{abstract}

Keywords: brain MRI; fuzzy c-means clustering; image segmentation; structural similarity measurement

\section{Introduction}

Brain disease is one of the principal diseases menacing human health nowadays. Utilizing the brain imaging techniques to analyze its function quantitatively is an important help for the effective diagnosis of brain disease. Magnetic resonance (MR) image acquisition is a medical imaging technique used in radiology to display internal structures of the body in detail. It possesses good contrast resolution for different tissues and is widely used in medical imaging technique. Reliable quantitative analysis of MR images can be performed by using image segmentation.

Image segmentation is defined as the partitioning of an image into non-overlapped, consistent regions, each with distinct characteristics, such as intensity, texture or color. Fuzzy segmentation methods are of considerable benefits, owing to the uncertainty of MR image. In particular, the transitional regions between tissues are unclear and their memberships are intrinsically vague [1]. Fuzzy c-means (FCM) clustering algorithm [2] is the best known and powerful method in fuzzy segmentations methods and its success chiefly attributes to the introduction of fuzziness for the belongingness of each image pixels. Unfortunately, MR images always contain uncertainly and unknown noise evoked by imaging mechanism. There is no consideration of spatial information in standard FCM clustering algorithm, so it is very sensitive to noise.

Recently, many researchers have introduced the spatial information derived from the image into the standard FCM to overcome the impact of noise in the medical images. Ahmed et al., [3] modified the objective function of FCM by incorporating spatial neighborhood term to allow the labeling of a pixel to be influenced by the labels in its immediate neighborhood and called the proposed algorithm as FCM_S. However, it is 
very time-consuming because of computing the neighborhood term in each iteration step. In order to reduce the loads of computation of FCM_S, Chen and Zhang [4] presented two variants: FCM_S1 and FCM_S2. These two algorithms substituted the neighborhood term of FCM_S with the mean-filtered image and median-filtered image, which can be computed in advance. But the selection of parameter that controls the balance between the original image and its corresponding mean- or median-filtered image has proved to be difficult [5].

It is known that an image patch contains more information than a single pixel and can better describe the characters of the image. Therefore image patches have been widely used in image denoising, especially for the algorithms based on non-local information [6, 7]. Zhao et al., [8] proposed FCM_NLS model by introducing non- local spatial constraints term into the objective function of FCM. This method need choose different parameters of the regular energy term when segmenting different images, and it is essentially isotropic, so that it would lose the structure information easily.

In this paper, we propose a novel fuzzy c-means model for brain MRI segmentation. Unlike the existing extensions of FCM algorithm, our method incorporates two influential factors. One is the non-local information determined by pixels whose neighborhood configurations look like the neighborhood of the pixel of interest; the other is the spatial structural similarity (SSIM) $[9,10]$ to extract structure information from image patches sufficiently. Consideration of the two constraints can effectively restrain the noise in the image and preserve more structure information as shown in our experiments.

The rest of this paper is organized as follows: In Section 2, the standard FCM algorithm and relevant extension are reviewed. Our proposed novel FCM model is described in Section 3. In Section 4, we present experimental and comparison results and conclude this paper in Section 5.

\section{Standard FCM Algorithm and Relevant Extension}

\subsection{Standard FCM Algorithm}

The standard FCM clustering algorithm is proposed by Dunn [11], and later improved by Bezdek [2]. It assigns a class membership to a data point, depending on the similarity of the data point to a particular class relative to all other classes. Utilizing the FCM algorithm to partition an image into $c$ clusters. Let $X=\left\{x_{1}, x_{2} \ldots x_{n}\right\}$ be an image with $n$ pixels, where $x_{k}$ represents the gray value of the $k$ th pixel. The standard FCM objective function is

$$
J_{m}(u, v)=\sum_{i=1}^{c} \sum_{k=1}^{n} \mu_{i k}^{m} d^{2}\left(x_{k}, v_{i}\right) \quad \text { subject to } \sum_{i=1}^{c} u_{i k}=1, u_{i k} \in[0,1]
$$

where $u_{i k}$ is the membership of the $k$ th pixel in the $i$ th cluster, and $v_{i}$ is the fuzzy cluster centroid of the $i$ th cluster. $d^{2}\left(x_{k}, v_{i}\right)=\left\|x_{k}-v_{i}\right\|^{2}$ measures the distance between $x_{k}$ and $v_{i}$, where $\|\cdot\|$ always use the Euclidean norm. $m$ presents the index of fuzziness (in our study, we choose $m=2$ ).

By minimizing Eq. (1) using the Lagrange multiplier method, the update equations of the membership function $u_{i k}$ and the cluster center $v_{i}$ are given as follows

$$
\mu_{i k}=\left(\sum_{j=1}^{c}\left(\frac{d\left(x_{k}, v_{i}\right)}{d\left(x_{k}, v_{j}\right)}\right)^{2 /(m-1)}\right)^{-1}
$$




$$
v_{i}=\frac{\sum_{k=1}^{n} \mu_{i k}^{m} x_{k}}{\sum_{k=1}^{n} \mu_{i k}^{m}}
$$

The FCM converges to a solution by iterating the two necessary conditions. Each pixel will be associated with a membership value for each class. Assigning the pixel to the class with the highest membership value, a segmentation of the image can be obtained.

The standard FCM is very sensitive to noise in the image, because the objective function does not take any spatial information into consideration. So we should improve the robustness to noise for the FCM algorithm by dealing with spatial context properly.

\subsection{FCM_S and its Variants}

In order to overcome this problem, Ahmed et al., [3] introduced a local spatial constraint term derived from the image into objective function of FCM (FCM_S). The improved objective function of FCM_S is expressed as

$$
J_{m}=\sum_{i=1}^{c} \sum_{k=1}^{n} \mu_{i k}^{m} d^{2}\left(x_{k}, v_{i}\right)+\frac{\alpha}{N_{R}} \sum_{i=1}^{c} \sum_{k=1}^{n} \mu_{i k}^{m} \sum_{r \in N_{k}} d^{2}\left(x_{r}, v_{i}\right)
$$

the second term is the spatial neighborhood term, in which $N_{k}$ denotes the set of neighbors falling into a window around the $k$ th pixel and $N_{R}$ is its cardinality. The parameter $\alpha$ controls the penalty effect of the spatial neighborhood term. The shortcoming is that computing the neighborhood term takes much time during each iteration step.

To reduce the computational complexity of FCM_S, Chen and Zhang [4] proposed two variants of FCM_S, and the objective function can be written as follows:

$$
J_{m}=\sum_{i=1}^{c} \sum_{k=1}^{n} \mu_{i k}^{m} d^{2}\left(x_{k}, v_{i}\right)+\alpha \sum_{i=1}^{c} \sum_{k=1}^{n} \mu_{i k}^{m} d^{2}\left(\bar{x}_{k}, v_{i}\right)
$$

where $\bar{x}_{k}$ is a mean or median of neighboring pixels lying within a window around $x_{k}$. $\bar{x}_{k}$ can be computed in advance, thus computational time can be saved in the clustering process. However, there exists a parameter $\alpha$ which controls the influence of spatial neighborhood term has proved to be difficult to select.

\section{Proposed Method}

In this section, a novel fuzzy C-means model based on the spatial similarity information is proposed to overcome the drawback of the standard FCM and some other related extensions. We replace each pixel used in constructing the objective function of FCM with the corresponding image patch, and all patches are weighted adaptively. Our proposed model views each image patch, instead of each pixel, so that spatial information is incorporated intrinsically into the segmentation process. The traditional patch model is isotropic, so that it would lose the structure information easily. Our method incorporates the non-local information and SSIM to be anisotropy and preserve more structure information.

It is common knowledge that the FCM-based image segmentation result is decided by the membership value. The membership value is decided by the distance measurement $d^{2}$ in Eq. (2). So we can infer that the distance measurement is the key to segmentation success. In our model, a new distance function is established by non-local information and SSIM.

\subsection{Structural Similarity (SSIM) Measurement}


The structural similarity (SSIM) index [9] is proposed by Zhou Wang et. al., to measure image similarity. It has shown superior performance over signal to noise ratio (SNR) and peak signal to noise ratio (PSNR). In SSIM theory, natural image are of highly structured, adjacent pixels have strong inter-dependencies, and these dependencies carry important information about the structure of the image.

The SSIM metric is calculated between two patches $x$ and $y$ of common size $N \times N$ is

$$
\operatorname{SSIM}(x, y)=\frac{\left(2 \mu_{x} \mu_{y}+c_{1}\right)\left(2 \sigma_{x} \sigma_{y}+c_{2}\right)}{\left(\mu_{x}^{2}+\mu_{y}^{2}+c_{1}\right)\left(\sigma_{x}^{2}+\sigma_{y}^{2}+c_{2}\right)}
$$

where $\mu$ is the average, $\sigma^{2}$ is the variance, and $\sigma_{x y}$ is the covariance of $x$ and y. $c_{1}$ and $c_{2}$ are two variables to stabilize the division with weak denominator, which are defined as

$$
c_{1}=\left(k_{1} L\right)^{2}, c_{2}=\left(k_{2} L\right)^{2}
$$

where $L$ is the dynamic range of the pixel-values. $k_{l}=0.01$ and $k_{2}=0.03$ are selected in our study.

The larger the value of SSIM is, the higher the similarity of two patches.

\subsection{Algorithm}

The proposed model takes the SSIM into the weight measurement between the adjacent patches to establish a new distance function. The new distance function between $x_{k}$ and $v_{i}$ is given as follows

$$
D^{2}\left(x_{k}, v_{i}\right)=\sum_{x_{j} \in N_{k}} w\left(x_{j}, x_{k}\right) d^{2}\left(x_{j}, v_{i}\right)
$$

where $N_{k}$ denote a search window of fixed size with respect to a center pixel $x_{k}$. The weight function is defined as

$$
w\left(x_{j}, x_{k}\right)=\left(1-\lambda_{k}\right) w_{s s i m}\left(x_{j}, x_{k}\right)+\lambda_{k} w_{n l}\left(x_{j}, x_{k}\right)
$$

Here we set $P_{k}$ denote a patch of fixed size with respect to a center pixel $x_{k}$, as same for $P_{j}$ to $x_{j}$. Then the weight influenced by SSIM is presented as

$$
w_{s s i m}\left(x_{j}, x_{k}\right)=\frac{\tilde{w}_{s s i m}\left(x_{j}, x_{k}\right)}{R\left(x_{k}\right)}
$$

where $\tilde{w}_{s s i m}\left(x_{j}, x_{k}\right)$ represents the SSIM-weight between $P_{k}$ and $P_{j} . R\left(x_{k}\right)$ is sum of SSIM-weight in $N_{k}$. In fact, the resultant SSIM value is a decimal value between -1 and 1 , so we set the SSIM-weight as

$$
\begin{aligned}
& \tilde{w}_{s s i m}\left(x_{j}, x_{k}\right)=e^{\frac{\left(2 \mu_{P_{j}} \mu_{P_{k}}+C_{1}\right)\left(2 \sigma_{P_{j} P_{k}}+C_{2}\right)}{\left(\mu_{P_{j}}^{2}+\mu_{P_{k}}^{2}+C_{1}\right)\left(\sigma_{P_{j}}^{2}+\sigma_{P_{k}}^{2}+C_{2}\right)}} \\
\text { and } \left.\quad x_{k}\right) & =\sum_{x_{j} \in N_{k}} \tilde{w}_{s s i m} x_{j} x_{k}
\end{aligned}
$$

In addition, the weight function influenced by non-local information $[6,7]$ is given as follows

$$
w_{n l}\left(x_{j}, x_{k}\right)=\frac{1}{Z\left(x_{k}\right)} U\left(x_{j}, x_{k}\right)
$$


where $U\left(x_{j}, x_{k}\right)$ is the exponential form of the similarity, and $Z\left(x_{k}\right)$ is the normalizing constant

$$
\begin{aligned}
& U\left(x_{j}, x_{k}\right)=e^{-\frac{\left\|v\left(P_{j}\right)-v\left(P_{k}\right)\right\|_{2, a}^{2}}{h^{2}}} \\
& Z\left(x_{k}\right)=\sum_{x_{j} \in N_{k}} e^{-\frac{\left\|v\left(P_{j}\right)-v\left(P_{k}\right)\right\|_{2, a}^{2}}{h^{2}}}
\end{aligned}
$$

The similarity between two pixels $x_{k}$ and $x_{j}$ depends on the similarity of the intensity gray level vector $v\left(N_{k}\right)$ and $v\left(N_{j}\right)$. The similarity is measured as a decreasing function of the weighted Euclidean distance $\left\|v\left(P_{j}\right)-v\left(P_{k}\right)\right\|_{2, a}^{2}$, where $\alpha$ is the standard deviation of the Gaussian kernel. The parameter $h$ is a degree of filtering to control the decay of the exponential function.

Finally, the tradeoff parameter of two weights is defined as

$$
\lambda_{k}=\max _{j \in N_{k}}\left(w_{n l}\left(x_{j}, x_{k}\right)\right)
$$

Then new distance function has been defined, the update equations of the membership function and the cluster center are given as follows

$$
\begin{gathered}
\mu_{i k}=\left(\sum_{j=1}^{c}\left(\frac{D\left(x_{k}, v_{i}\right)}{D\left(x_{k}, v_{j}\right)}\right)^{2 /(m-1)}\right)^{-1} \\
v_{i}=\frac{\sum_{k=1}^{n} \mu_{i k}^{m} x_{k}}{\sum_{k=1}^{n} \mu_{i k}^{m}}
\end{gathered}
$$

We can describe our proposed model as below

Step 1: Set the number of clusters c. Initialize the fuzzy cluster centroid vector $V=\left[v_{l}\right.$,

$\left.v_{2} \ldots v_{c}\right]$ by k-means clustering algorithm and set $\varepsilon>0$ to a very small value.

Step 2: Set the patch size and search window size.

Step 3: Calculate $w\left(x_{j}, x_{k}\right)$ using Eq. (9).

Step 4: Update $D^{2}\left(x_{j}, x_{k}\right)^{(t+1)}$ using Eq. (8).

Step 5: Update $\mu_{i k}^{(t+1)}$ using Eq. (17).

Step 6: Update $v_{i}^{(t+1)}$ using Eq. (18).

Step 7: Repeat Steps 4-6 until satisfy the termination criterion $\|\boldsymbol{V}(t+1)-V(t)\|<\varepsilon$.

\section{Experimental Results and Analysis}

Our model was applied in MATLAB on a PC with Intel Pentium IV 2.4G processor and 1024 RAM. In order to test the reliability and validity of the algorithm, we execute the segmentation on both synthetic and real MR brain images from the McConnell Brain Imaging Center at the Montreal Neurological Institute, McGill University, and it provides perfect segmentation results of synthetic brain images. In our model, we set the parameters as: $c=4, \mathrm{~h}=16, \varepsilon=10^{-5}$, the radius of search window is 5 , and the radius of patch is 3 .

\subsection{Segmentation of Synthetic Brain MR Images}

In Figure 1, the first row has three initial images with noise level 3\%, 5\% and 7\%, we can see that noise with different degrees of increase in the initial images. The second row 
is segmentation results by FCM method, we can find that due to the effect of the noise, many wrong results appear, and the more the impact of noise, the worse the segmentation results. Because the standard FCM does not take any spatial information into consideration. The third and fourth row are segmentation results by FCM_S1 and FCM_S2, the two methods have better results than FCM by using spatial information. When the initial images are less affected by noise, good segmentation results can be obtained. However, when images are more affected by noise, many wrong results appear, especially on the white matter. It can be concluded that the two methods are not robust to the noise. The fifth row is segmentation results by FCM_NLS, this method can be robust to noise, and obtain better results than FCM, FCM_S1 and FCM_S2. But some wrong results by excessive smooth appear on the boundaries between tissues. It is because the model is isotropic, so that it would lose the structure information easily. The sixth row is results by our proposed model, this method can not only be robust to noise, but also preserve the slender structure effectively, since our model take both the non-local information and spatial structural similarity measurement (SSIM) between the image patches into consideration to improve the robustness to noise and preserve more structure information.
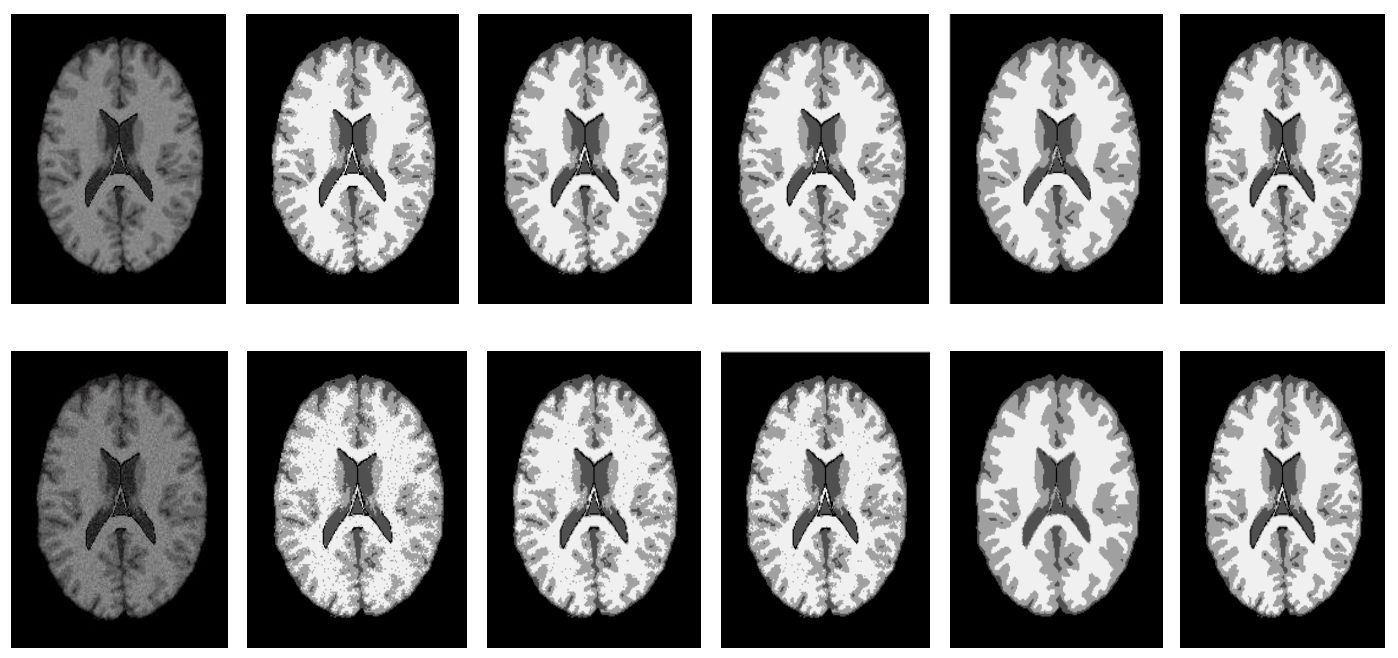

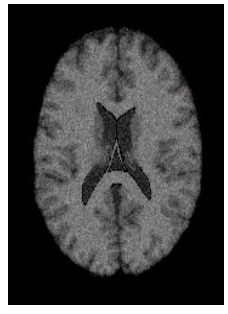

(a)

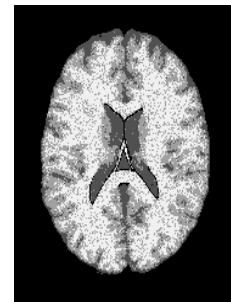

(b)

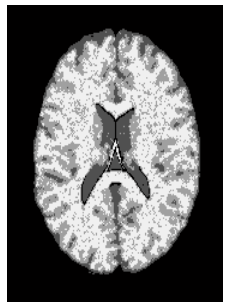

(c)

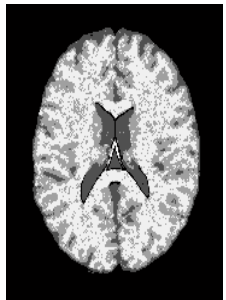

(d)

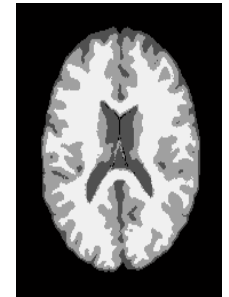

(e)

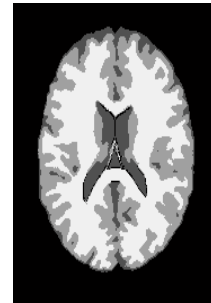

(f)

Figure 1. Segmentation of the Synthetic Brain MR Images: (a) Original Image;

(b) Result by FCM Method; (c) Result by FCM_S1 Method; (d) Result by FCM_S2 Method; (e) Result by FCM_NLS Method; (f) Result by our Method

In order to quantitatively evaluate the benefits, we segment 20 sets of synthetic brain images with different levels of noise. We use the Jaccard similarity [12] (JS) index to estimate the accuracy of segmentation.

$$
J\left(S_{1}, S_{2}\right)=\frac{\left|S_{1} \cap S_{2}\right|}{\left|S_{1} \cup S_{2}\right|}
$$

where $S_{l}$ is the ground truth, and $S_{2}$ is the segmentation result. A good algorithm would give high JS values. The average quantitative results of white matter (WM) and gray 
matter (GM) are listed in Table 1. It can be seen that our method is more accurate than others.

Table 1. Evaluation of Tissue Segmentation in Terms of Jaccard Similarity Coefficients (\%)

\begin{tabular}{ccccccc}
\hline noise level & Tissue & FCM & FCM_S1 & FCM_S2 & FCM_NLS & our method \\
\hline \multirow{2}{*}{$3 \%$} & WM & 92.61 & 92.90 & 93.52 & 92.68 & 93.19 \\
\cline { 2 - 7 } & GM & 86.40 & 85.41 & 87.50 & 87.45 & 87.97 \\
\hline \multirow{2}{*}{$5 \%$} & WM & 82.56 & 85.74 & 86.93 & 90.50 & 92.03 \\
\cline { 2 - 7 } & GM & 71.79 & 75.64 & 77.53 & 81.61 & 85.42 \\
\hline \multirow{2}{*}{$7 \%$} & WM & 69.17 & 73.56 & 72.41 & 84.78 & 89.49 \\
\cline { 2 - 7 } & GM & 55.73 & 61.32 & 60.32 & 75.23 & 80.68 \\
\hline
\end{tabular}

\subsection{Segmentation of Real MR Brain Image}

To illustrate the benefit of our method further, Figure 2 shows the segmentation result of real MR brain image. Figure 2 (a) is the original real MR brain image, noise and slender structure are contained in it. Figure 2 (b) is the result by standard FCM. Wrong segmentation results appear due to the impact of noise. Figure 2 (c), (d) and (e) are results by FCM_S1, FCM_S2, and FCM_NLS. This three model can reduce the impact of noise, but they can not preserve the slender structural information effectively Figure 2 (f) is result by our proposed method. It can be observed that our method can obtain more accurate segmentation results than others, especially on the slender structure of white matter.

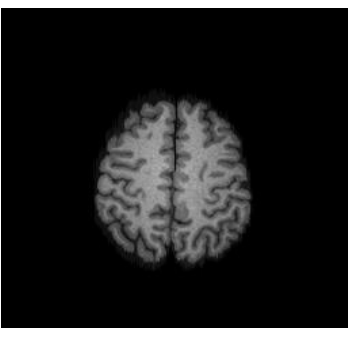

(a)

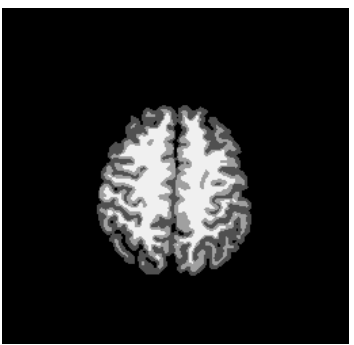

(d)

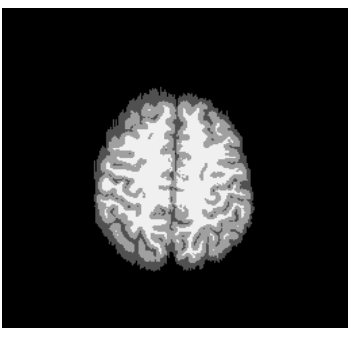

(b)

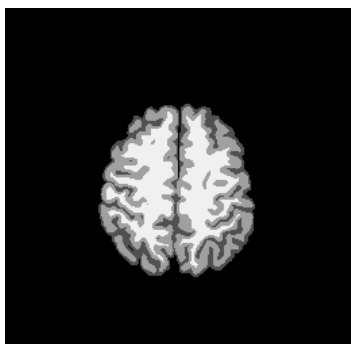

(e)

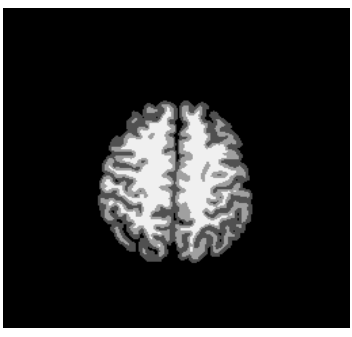

(c)

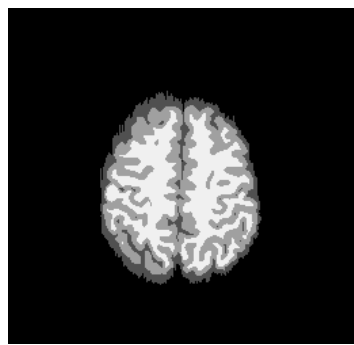

(f)

Figure 2. Segmentation Results of Real MR Brain Image: (a) Original Image;

(b) Result by FCM Method; (c) Result by FCM_S1 Method; (d) Result by FCM_S2 Method; (e) Result by FCM_NLS Method; (f) Result by our Method 


\section{Conclusion}

In this paper, we have presented a novel fuzzy C-means model based on the spatial similarity information. The method was formulated by modifying the distance function to compensate for noise by using both the non-local information and SSIM. Experimental results have shown that our method can outperform other segmentation methods for the images with noise.

\section{Acknowledgements}

This research work was supported by the National Nature Science Foundation of China (61003209, 61173072), and the Natural Science Foundation of Jiangsu Province (BK2011824, BK2012461).

\section{References}

[1] C. M. Li, C. Y. Xu and A. W. Anderson, "MRI tissue classification and bias field estimation based on coherent local intensity clustering: a unified energy minimization framework", Proceedings of Information Processing in Medical Imaging. Berlin: Springer, vol. 21, (2009).

[2] J. Bezdek, "Pattern recognition with fuzzy objective function algorithms", Norwell: Kluwer Academic Publishers, (1981), pp. 215-220.

[3] M. Ahmed, S. Yamany, N. Mohamed, A. Farag and T. Moriarty, "A modified fuzzy C-means algorithm for bias field estimation and segmentation of MRI data", IEEE Transactions on Medical Imaging, vol. 21 , no. 3, (2002).

[4] S. Chen and D. Zhang, "Robust image segmentation using FCM with spatial constraints based on new kernel induced distance measure", IEEE Transactions on Systems, Man and Cybernetics, vol. 34, no. 4, (2004).

[5] W. L. Cai, S. C. Chen and D. Q. Zhang, "Fast and robust fuzzy c-means clustering algorithm incorporating local information for image segmentation", Pattern Recognition, vol. 40, no. 3, (2007).

[6] A. Pardo, "Analysis of non-local image denoising methods", Pattern Recognition Letters, vol. 32, no. 16, (2011).

[7] Y. L. Liu, J. Wang, X. Chen, Y. W. Guo and Q. S. Peng, “A robust and fast non-local means algorithm for image denoising", Journal of Computer Science and Technology, vol. 23, no. 2, (2008).

[8] F. Zhao, L. C. Jiao and H. Q. Liu, "Fuzzy c-means with non local spatial information for noisy image segmentation", Front. Comput. Sci. China, vol. 5, no. 1, (2011).

[9] Z. Wang, A. C. Bovik, H. R. Sheikh and E. P. Simoncelli, "Image quality assessment: from error visibility to structural similarity", IEEE Transactions on Image Processing, vol. 13, no. 4, (2004).

[10] A. Rehman and Z. Wang, "Reduced-Reference Image Quality Assessment by Structural Similarity Estimation", IEEE Transactions on Image Processing, vol. 21, no. 8, (2012).

[11] J. C. Dunn, "A fuzzy relative of the ISODATA process and its use in detecting compact well separated cluster", Journal of Cybernet, vol. 3, no. 3, (1974).

[12] U. Vovk, F. Pernus and B. Likar, "A review of methods for correction of intensity inhomogeneity in MRI", IEEE Transactions on Medical Imaging, vol. 26, no. 3, (2007).

\section{Authors}

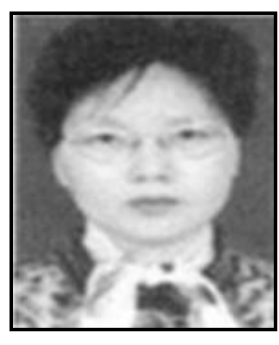

Shunfeng Wang, Professor at the School of BinJiang, Nanjing University of Information Science and Technology. Her research interest covers image processing, pattern recognition.

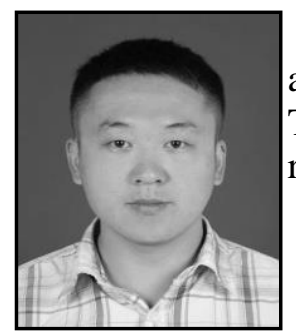

Zhiyuan Geng, Graduate student at the College of Mathematics and Statistics, Nanjing University of Information Science and Technology. His research interest covers image processing, pattern recognition. 


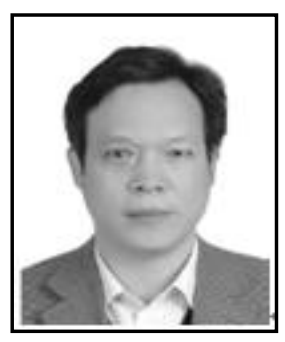

Jianwei Zhang, Dr. Jianwei Zhang is a Professor at the College of Mathematics and Statistics, Nanjing University of Information Science and Technology. His research interest covers pattern recognition, artificial intelligence, and remote sensing information processing.

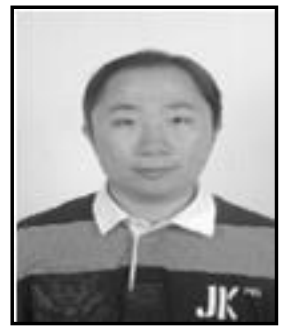

Yunjie Chen, Dr. Yunjie Chen received the B.S. and M.S. degree in the Applied Mathematics from Nanjing University of Information Science and Technology, China in 2002 and 2005, respectively. He received $\mathrm{Ph}$.D. degree in Pattern Recognition and Intelligent Systems from NUST. Now, he is an associate professor in NUIST. His research interests include image processing, pattern recognition and numerical analysis.

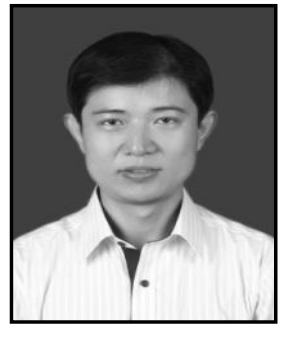

Jin Wang, Dr. Jin Wang received the B.S. and M.S. degree in the Electronical Engineering from Nanjing University of Posts and Telecommunications, China in 2002 and 2005, respectively. He received Ph.D. degree in the Ubiquitous Computing laboratory from the Computer Engineering Department of Kyung Hee University Korea in 2010. Now, he is a professor in the Computer and Software Institute, Nanjing University of Information Science and technology. His research interests mainly include routing method and algorithm design, performance evaluation and optimization for wireless ad hoc and sensor networks. He is a member of the IEEE and ACM. 
International Journal of Signal Processing, Image Processing and Pattern Recognition Vol.7, No.1 (2014) 